\title{
15 years of acoustic detection studies at INFN
}

\author{
Salvatore Viola ${ }^{1, \star}$ and Giorgio Riccobene ${ }^{1}$ \\ ${ }^{1}$ INFN - Laboratori Nazionali del Sud
}

\begin{abstract}
Since the early 2000s, thanks to national, regional and European projects, a number of abyssal detectors equipped with acoustic sensors have been installed and operated by INFN in two cabled submarine research infrastructures off Eastern Sicily. The INFN's interest in underwater acoustics arises from the need to develop an acoustic positioning system for the $\mathrm{KN} 3 \mathrm{NeT}$ telescope and to study the possibility of neutrino acoustic detection. The use of innovative technologies for data acquisition and transmission systems have enabled the first long-term studies on the deep marine environment of the Ionian Sea with a variety of lines of research. Data acquired through the INFN underwater infrastructures have allowed the continuous monitoring of the underwater acoustic noise and several studies on cetacean species present in the area and on seismic sources . An overview of the main activities of INFN on detection and localization of underwater acoustic sources will be presented.
\end{abstract}

\section{Introduction}

The interest in underwater acoustics at Istituto Nazionale di Fisica Nuclere (INFN) began in the early 2000's with the first studies on the design of an underwater Cherenkov neutrino telescope to be installed in the Mediterranean Sea. In particular studies on underwater acoustics carried on by INFN have been addressed to the development of an acoustic positioning system for the telescope infrastructures and at the measurement of the underwater background noise for very high energy neutrino detection through thermo-acoustic technique. At that time underwater ambient noise was well characterized by military studies at surface but very few data in deep sea were available.

\section{NEMO-O $v$ DE: Ocean Noise Detection Experiment}

NEMO- $\mathrm{O} v \mathrm{DE}$ was the first experiment to perform long-term measurement and monitoring of the acoustic background at $2100 \mathrm{~m}$ depth. It was installed in January 2005 at $25 \mathrm{~km}$ off Catania, in the East coast of Sicily (Ionian Sea), taking advantages of the underwater and terrestrial infrastructures developed for the NEMO (NEutrino Mediterranean Observatory) project. NEMO- OvDE was a cabled array of 4 large bandwidth hydrophones mounted in a tetrahedral configuration (1 $\mathrm{m}$ size). The acoustic antenna was powered from shore at 220 VAC. The four hydrophones, model Reson TC4042, have been individually calibrated from $5 \mathrm{kHz}$ to $90 \mathrm{kHz}$ in sensitivity and directivity. All analogue acoustic signals were digitized off-shore through a low noise 4 channel audio ADC system $(96 \mathrm{kHz}$,

^e-mail: sviola@lns.infn.it 


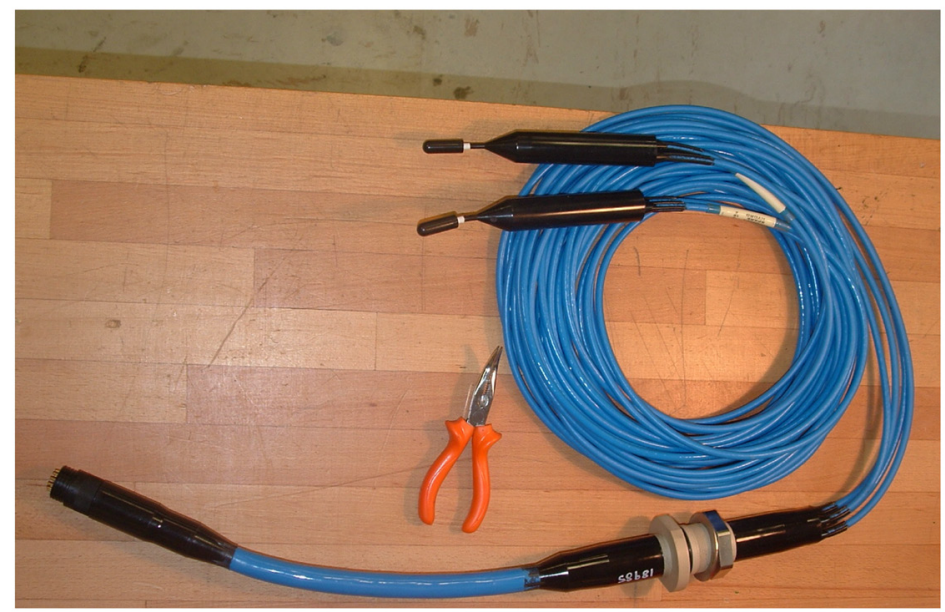

Figure 1. Couple of large bandwidth hydrophones developed by SMID for SMO project.

24 bit sampling, $\Delta \Sigma$ ) and sent to shore on optical fibres. Data coming from the 4 synchronized acoustic channels have been stored in WAV format with a duty cycle of $5 \mathrm{~min} /$ hour from January 2005 to November 2006 [1]. Studies of acoustic background levels and their variations as a function of time and correlation with weather conditions, seismic, biological and anthropogenic sources have been carried on. NEMO- $\mathrm{O} v \mathrm{DE}$ data represent first long-term ambient noise measurement at very deep sea in Mediterranean Sea. Acquired data have been shared with marine biology community for important studies on marine mammals in the area (sperm whales [2] and dolphins [3]).

\section{SMO-Submarine Multidisciplinary Observatory}

In 2013 in the framework of SMO (Submarine Multidisciplinary Observatory) project a 3D array of 14 large bandwidth acoustic sensors has been installed on board the Cherenkov neutrino detector prototype NEMO - Phase II. The array was deployed at depth of $3500 \mathrm{~m}$, about $100 \mathrm{~km}$ off-shore South-East Sicily. Goals of the projects were the deep-sea test of a novel acoustic positioning system for a $\mathrm{km}^{3}$-scale underwater neutrino telescope. Long term and real-time monitoring of high frequency acoustic background at different depths and development of new technologies for the detection of high energy particle interactions in water. INFN and the Italian company SMID have developed for SMO project a low cost hydrophone for $4000 \mathrm{~m}$ depth, with no change of sensitivity as a function of depth (Fig.1).

SMO data acquisition chain was designed in order to synchronize all acoustic sensors with an uncertainty better than $1 \mu \mathrm{s}$. Analog signals from the 14 hydrophones were sampled offshore at 192 $\mathrm{kHz}$ with a resolution of 24 bit by 7 professional audio stereo ADCs, driven by a single Master Clock located on shore. Digital data were labelled in situ with time stamps distributed by a GPS receiver on shore and sent to shore thanks to point to point optical connections [5]. A main challenge of the project was the time calibration of array. Electronics boards' latency, data transmission delay on optical fibres and hydrophone' s ceramics latency have been separately measured. All of these components 
participate to the time accuracy of each acquisition channel. In situ measurements demonstrated optimal performance in terms of frequency and time resolution. The analysis of the acoustic signals at $32 \mathrm{kHz}$ from external beacons of the positioning system of the detector experimentally confirmed the nominal sensitivity of the sensors at $3500 \mathrm{~m}$ water depth.

The continuous stream of acoustic data from each hydrophone was analysed in real time for positioning purposes and, every hour, 5 minutes of unbiased raw data were stored on hard disks and saved in a digital library for off-line studies. Impulsive and continuous sounds have been monitored from March 2013 to August 2014.

\section{O $v$ DE 2}

The technology developed for the acoustic antenna SMO was applied to a smaller array installed in 2012 at the same location of the previous NEMO-OvDE experiment. The array is fully operative since February 2017. Signals acquired by 4 large bandwidth frequency hydrophones are digitized by 2 synchronised stereo ADCs (192kHz/24 bit). Thanks to the data acquisition architecture designed by INFN for $\mathrm{O} v \mathrm{DE} 2$, acoustic data streams are dispatched through TCP/IP protocol to a computer farm to be continuously analyzed in real time. The direct optical fibre high speed connection from deep-sea to a GARR (Italian academic and research network) node, make it possible the on-line analysis of the acoustic data stream by machines spread all over the world.

The underwater background noise is continuously monitored by calculating the acoustic average Power Spectral Density every second. Maximum, $90^{\text {th }}$ percentile, $50^{\text {th }}$ percentile, $10^{\text {th }}$ percentile and mean values are computed for each frequency bin in $5 \mathrm{~min}$ long time windows. Spectrogram, percentile values and plots related to 5 minutes long acquisitions are stored in a digital library. Fig. 2 reports an example of the plots in JPEG format produced every 5 minutes by the on-line software devoted to monitoring of the background acoustic noise.

Like in SMO array, raw data acquired by each hydrophone are also saved in a storage with a duty cycle of 5 minutes per hour for further off-line analysis.

\section{NEMO-SN1}

Thanks to the knowledge acquired by INFN in the development of instrumentation for underwater measurements, INFN was also involved in the construction and operation of the multidisciplinary observatory NEMO-SN1. NEMO-SN1 is the first-established node of EMSO (European Multidisciplinary Seafloor Observatory). The observatory was in operation from 2012 to 2013 . It hosted seismic and oceanographic instrumentation and acoustic sensors for the study of the underwater environment. An acoustic array of 4 hydrophones was devoted to studies at high frequencies. It is composed of 4 SMID large bandwidth calibrated hydrophones, of the same model used in SMO and OvDE 2 projects. Acquired signals are sampled off-shore by a commercial four-channels audio analog-to-digital converter and time-stamped on shore by a dedicated electronic board.

A seismic hydrophone, model SMID DT405D, calibrated in sensitivity within the frequency range 1-1000 Hz, was used to monitor the background low frequency noise. Analog signals acquired by the seismic hydrophone have been continuously sampled at $2 \mathrm{kHz}$, saved in 10 minutes long files and stored at the INFN - LNS. Mean values of PSD in each recording have been calculated and correlated to ship traffic in the area. Studies performed by INFN on environmental low frequency signals have also concerned acoustic noise induced by seismic airgun surveys and effects of acoustic pollution on underwater fauna. In particular, the acoustic presence of fin whale in the Gulf of Catania was studied in relation to noise levels in its emission frequency band [6] [7]. 

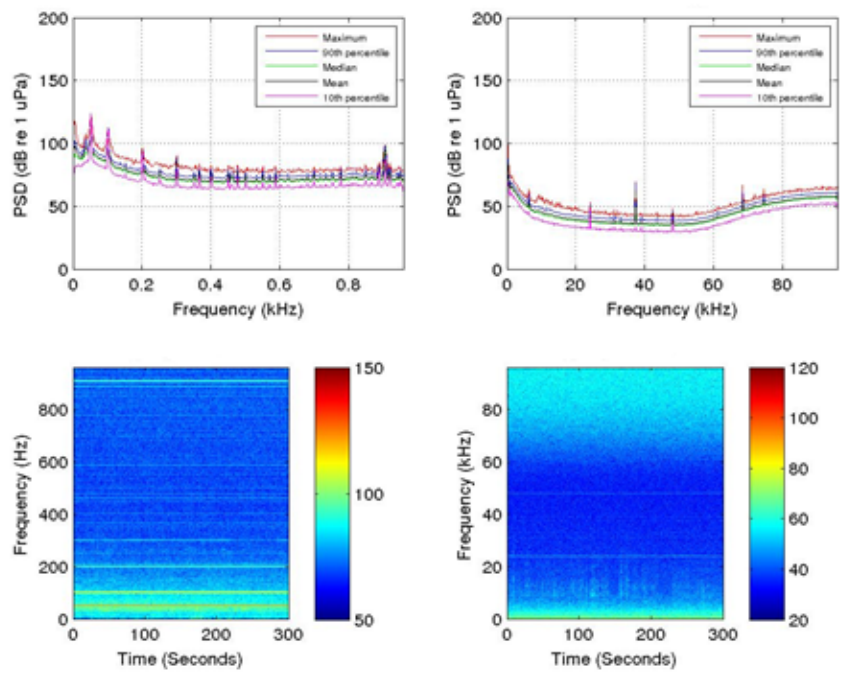

Figure 2. Example of plots produced every 5 minutes by the on-line software devoted to monitoring of the background acoustic noise. Plots include spectrograms and different PSD percentiles in 0-1 kHz range ad 0-96 $\mathrm{kHz}$ range and are archived in JPEG format for rapid off-line visualization.
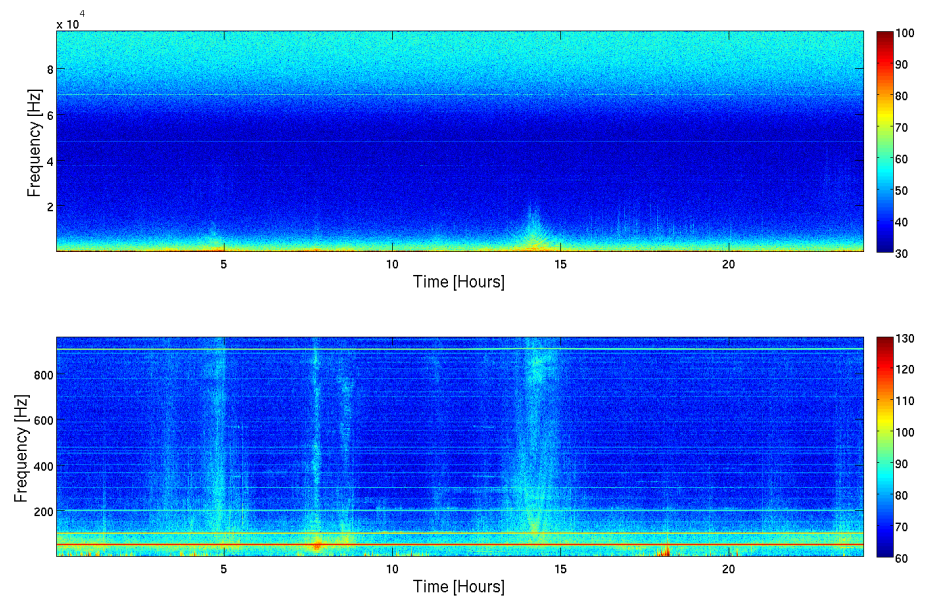

Figure 3. Typical daily spectrogram obtained by merging the output of on-line software for continuous background noise monitoring in $0-1 \mathrm{kHz}$ (top) and $0-96 \mathrm{kHz}$ (down) frequency ranges. 


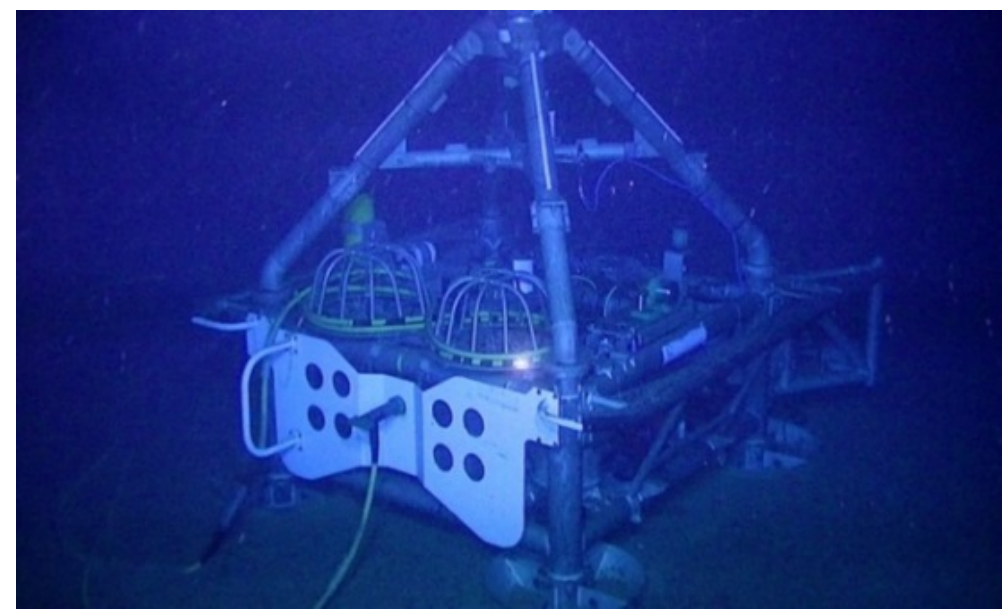

Figure 4. Picture of the NEMO-SN1 multidisciplinary observatory at the INFN underwater site, in the Gulf of Catania (2100 m water depth).

\section{Monitoring of underwater transient signals}

Neutrino detection through thermo-acoustic technique needs an accurate knowledge of the acoustic environment not only in terms of continuous diffuse noise. The expected signals produced by a very high energy neutrino interaction in sea water is a bipolar acoustic pulse in the frequency range $10 \div$ $40 \mathrm{kHz}$. At these frequencies, transient signals coming from biological sources (dolphin and sperm whale echolocation clicks) and anthropogenic sources (ship cavitation,sonars, pingers, ...) represent a constant background noise for the neutrino detection. Lack of information on transient signals in deep sea has pushed INFN to collaborate with different scientific communities (biology, geology, ocenography,...) for a systematic study of the underwater transient noises through a multidisciplinary approach. One of the most frequent transient signals found in INFN recordings is the sperm whale echolocation click in the frequency range $3 \div 20 \mathrm{kHz}$. Sperm whale clicks have been detected in 50 $\%$ of the recording days in 2005 and $46 \%$ of the recording days in 2006.

Automatic detection software have been developed to recognize sperm whale clicks in already existing recordings or by analyzing data stream in real time. For each detection, click waveforms acquired by all hydrophones are extracted from the stream ( $40 \mathrm{~ms}$ around the detection time), labeled with GPS time of acquisition and made available for further analysis saving space in the storage.

Dolphins' clicks represent an almost continuous background for the neutrino acoustic detection at frequencies higher than $25 \mathrm{kHz}$. Indeed, dolphins' clicks have been detected in $79 \%$ of the recordings acquired in 2005 and $87 \%$ in 2006. Different species of dolphins are present in the Ionian Sea. Algorithms to investigate presence of dolphin vocalizations, both clicks and whistles, have been also developed and compared with manual detection performed by human-operators. The long term monitoring of the echolocation activity in the Ionian Sea allowed the discovery of diel patterns [3].

Preliminary studies for the development of a neutrino signal trigger are on-going. INFN is working on methods to discard possible sources of false detections by using real background noise dataset. The vast catalogue of impulsive sounds acquired in deep sea by INFN underwater infrastructures 
represents a import tool to test and instruct detection algorithms for discerning candidate neutrino events from biological and athropogenic transient signals.

\section{Conclusions}

INFN started dealing with underwater acoustics in the early 2000's. In more than 15 years of activities on acoustics in deep water, INFN has developed new technology and instrumentation for the detection of very high energy neutrino events through thermo-acoustic technique.

An innovative data acquisition architecture, based on "all data to shore" philosophy, has been developed e successfully validated at $3500 \mathrm{~m}$ water in the framework of SMO project. This allowed to entirely move on shore the processing of the acoustic signals saving underwater power consumption and making it possible a continuous improvement of the analysis software without intervention on underwater instrumentation.

Most of the technological solutions developed by INFN for SMO are now used in the acoustic positioning system of KM3NeT neutrino telescope. Acoustic data acquired by INFN since 2006 off Sicilian coasts represent, at present, the largest data-set of underwater sounds in the Mediterranean Sea.

The availability of these large datasets allows to develop new approaches on acoustic neutrino detection. Detection algorithms can be tested and validated in real conditions and detection sensitivity can be better evaluated by using in Montecarlo simulations real acoustic background noise measured at very deep sea. Furthermore, the technology developed for passive acoustic monitoring in deep waters has allowed the INFN to contribute to important scientific studies in the fields of marine biology, geology and ecology.

\section{References}

[1] G. Riccobene et al., Nuclear Instruments and Methods in Physics Research Section A: Accelerators, Spectrometers, Detectors and Associated Equipment 604, S149, (2009)

[2] Caruso F. et al., PLoS ONE, 10(12),(2015)

[3] Caruso F. et al., Scientific Reports, 7(1), 4321,(2017)

[4] Viola S. et al., Marine Pollution Bulletin, 121(1-2), 97-103, (2017)

[5] Viola S. et al., Nuclear Instruments and Methods in Physics Research Section A: Accelerators, Spectrometers, Detectors and Associated Equipment, 725,207-210, (2013)

[6] Sciacca V. et al.m PLoS ONE, 10(11),(2015)

[7] Sciacca V. et al., Acoustical Society of America ,Proceedings of Meetings on Acoustics 27,040010 (2017) 